\title{
Astragaloside IV Exerts Cardioprotection in Animal Models of Viral Myocarditis: A Preclinical Systematic Review and Meta-Analysis
}

\author{
Zhuang Zhuang ${ }^{1}$, Zi-Hao Wang ${ }^{1}$, Li-Hui Deng ${ }^{1}$, Qun Zheng ${ }^{1}$, Guo-Qing Zheng ${ }^{2 *}$ \\ and Yan Wang ${ }^{1 *}$ \\ ${ }^{1}$ Department of Cardiology, the Second Affiliated Hospital and Yuying Children's Hospital of Wenzhou Medical University, \\ Wenzhou, China, ${ }^{2}$ Department of Integrative Medicine of Neurology, the Second Affiliated Hospital and Yuying Children's \\ Hospital of Wenzhou Medical University, Wenzhou, China
}

OPEN ACCESS

Edited by:

Claudio Ferrante,

Università degli Studi G. d'Annunzio

Chieti e Pescara, Italy

Reviewed by:

Nasiara Karim,

University of Malakand, Pakistan

Satish Ramalingam,

SRM Institute of Science and

Technology, India

${ }^{*}$ Correspondence:

Guo-Qing Zheng

gq_zheng@sohu.com

Yan Wang

wywzchina@sina.com

Specialty section: This article was submitted to Inflammation Pharmacology, a section of the journal

Frontiers in Pharmacology

Received: 29 July 2019 Accepted: 31 October 2019 Published: 28 November 2019

Citation:

Zhuang Z, Wang Z-H, Deng L-H, Zheng $Q$, Zheng G-Q and Wang $Y$ (2019) Astragaloside IV Exerts

Cardioprotection in Animal Models

of Viral Myocarditis: A Preclinical Systematic Review and Meta-Analysis.

Front. Pharmacol. 10:1388.

doi: 10.3389/fphar.2019.01388
Astragaloside IV (AS-IV), the essential active component of astragalus, possesses diverse biological activities that have beneficial effects against cardiovascular disease. Here, we conducted a preclinical systematic review of 15 studies including 577 animals to establish the efficacy and potential mechanisms of AS-IV for animal models of viral myocarditis (VM). Six databases were searched from inception to October 2018. Application of the Cochrane Collaboration's tool 10-item checklist and Rev-Man 5.3 software to analyze risk of bias of studies and data on outcome measures revealed study quality scores ranging from 2 to 5 . Compared with the control group, AS-IV induced a marked decrease in mortality $(P<0.05)$, inflammation of myocardium and pathological score $(P<0.05)$ and cardiac enzymes expression $(P<0.05)$, and improved the function of the heart $(P<0.05)$. The potential mechanisms of AS-IV action were determined as antiremodeling of myocardium ( $n=1)$, anti-virus $(n=2)$, antioxidant $(n=2)$, anti-inflammatory $(n=6)$, anti-apoptosis $(n=1)$ and alleviation of myocardial fibrosis $(n=2)$. The collective results indicate that AS-IV exerts cardioprotective effects in animals with VM via multiple signaling pathways.

Keywords: Astragaloside IV, myocarditis, efficacy, mechanisms, meta-analysis

\section{INTRODUCTION}

Myocarditis is pathologically and clinically defined as an inflammatory disease of the heart muscle based on histological, immunological, and immunohistochemical criteria of cardiomyopathy classification from the World Health Organization (WHO) (Pollack et al., 2015). Myocarditis often arises from infection with common viruses, such as respiratory viruses and enteroviruses, and is most commonly associated with coxsackievirus B (Dennert et al., 2008). Myocarditis infection can be subdivided into three pathological stages (Mason, 2003). In the first stage, viral-mediated lysis directly destroys cardiomyocytes, leading to cardiomyocyte damage and cardiac dilation (McManus et al., 1993). The second stage is imbalance of immune regulation resulting from myocardial cell damage (Lawson, 2000). Finally, the typical manifestation of dilated cardiomyopathy 
(DCM) occurs due to extensive myocardial injury (Caforio et al., 2002). Viral myocarditis (VM) has the characteristics of myocardial inflammation, often developing into chronic dilated cardiomyopathy, acute myocarditis and even congestive heart failure (Kühl and Schultheiss, 2009). VM accounts for $12 \%$ of sudden deaths in patients under 40 years of age and is the leading cause of dilated cardiomyopathy, resulting in $50 \%$ of patients deaths within 1-2 years after diagnosis (Cooper et al., 2014). The incidence of myocarditis worldwide was reported a 22 cases per 100,000 individuals in 2013 , and about 1.5 million patients were diagnosed with myocarditis according to the ninth revised diagnosis of the International Classification of Diseases (GBD 2015 Disease and Injury Incidence and Prevalence Collaborators, 2016). For VM, antiviral drugs, physical activity restriction and myocardial nutrition are the main routine clinical treatments. Patients should follow the current guidelines for heart failure treatment in cases of left ventricular dysfunction or symptomatic heart failure (Ponikowski et al., 2016). However, establishing the potential benefits of immunomodulators and antiviral therapy is currently at the preliminary research stage (Pollack et al., 2015).

AS-IV, the essential active component of astragalus, has been traditionally used to treat various diseases. This compound is frequently reported to exert protective effects on cardiovascular, nervous and immune systems due to its antioxidant and activitiesantiapoptotic activities as well as regulatory effects on calcium balance (Ren et al., 2013). Systematic studies on animal models play an important role in drug research and provide substantial evidence to support application in the clinic (Sena et al., 2014). The purposes of the present study was to investigate the efficacy and potential mechanisms of action of AS-IV in animal models of VM.

\section{METHODS}

The study was carried out according to the Preferred Reporting Items for Systematic Review and MetaAnalyses (Stewart et al., 2015).

\section{Search Strategies}

Based on the literature retrieval from PubMed, Wanfang database, EMBASE, China National Knowledge Infrastructure (CNKI), Cochrane Library and VIP databases, animal experimental studies of AS-IV for VM were identified. All search strategies were performed from inception until October 2018. We selected the following terms: 1. Astragaloside; 2. Myocarditis; 3. 1 AND 2; 4. Animals NOT humans; 5. 3 AND 4.

\section{Eligibility Criteria}

Studies that met the following criteria were included: (1) animal models of VM were established through various ways, (2) treatment groups were administered any dose of AS-IV only, and comparators given non-functional and isasteric liquid (carboxymethyl cellulose or normal saline) or no treatment, and (3) the primary outcome measures were mortality rate, histopathological changes of myocardium, indicators of cardiac ultrasound, cardiac troponin, cardiac enzymes and/or level of ST-segment depression. Cardioprotective mechanisms of AS-IV action against VM were used as the secondary outcome measure. Exclusion criteria were as follows: (1) duplicate publication, (2) not in vivo studies, (3) comparison with other traditional Chinese medicine (TCM), (4) combinations with other medicine, and (5) lack of a control group.

\section{Data Extraction}

Two authors independently extracted the following details: (1) name of the first author and publication year, (2) characteristics (species, number, sex, and weight) of the animals used in each study, (3) methods to establish animal models of VM, (4) the therapeutic regimens of treatment and control groups, including method of administration, therapeutic drug dosage and sessions of treatment, (5) primary and secondary outcomes. If the results were obtained at different time-points or following administration of different doses of drug, only the final measured value and data obtained with the highest dose of drug were included. We attempted to contact authors for more information when published data for some records were only shown in a graphical format. In cases where no response was received, data in the graph were measured.

\section{Risk of Bias in Individual Studies}

The risk of bias was assessed by two independent authors through applying the ten-item scale (Hooijmans et al., 2014), with minor modifications. Risk of bias of the following domains were assessed: A: sequence generation; B: baseline characteristics; C: allocation concealment; D: random housing and other animal welfare; E: blinding of caregivers and/or investigators; F: random outcome assessment; G: blinding of outcome assessor; $\mathrm{H}$ : complete outcome data; I: selective outcome reporting; J: other sources of bias. One point was awarded for each item. Divergent findings between the two authors were settled through resolving by consensus or arbitration by the corresponding author.

\section{Statistical Analysis}

RevMan version 5.3 was utilized for statistical analysis. Standardized mean difference (SMD) instead of Mean differences (MDs) was employed as a summary statistic when data were not reported on the same scale. Heterogeneity and choice of effects models were investigated with the aid of standard chi-square test and $\mathrm{I}^{2}$ statistic test. Differences were considered significant at $\mathrm{P}$ values $<0.05$.

\section{RESULTS}

\section{Study Selection}

We identified a total of 540 hints on the basis of pertinent literature retrieval from the databases. After removing 439 reduplicated or irrelevant articles, 101 reports remained. Next, we excluded 53 non-animal studies through screening the titles and abstracts. Overall, 33 articles were excluded after reading the remaining full-text articles owing to: (1) no predetermined outcome index, (2) comparison with other TCM, (3) combination with other medicine, (4) non-viral myocarditis models, and (5) lack of a control group, leading to the final selection of 15 eligible articles (Zhang et al., 2003; Yu et al., 2005; Zhang et al., 2006; Wang and Li, 
2007; Luo et al., 2008; Liu et al., 2009; Luo et al., 2010; Chen et al., 2011; He and Li, 2011; Wang, 2012; Zhou et al., 2012; Liu et al., 2014; Gui et al., 2015; Tian et al., 2015; Xiao et al., 2016) (Figure 1).

\section{Characteristics of Included Studies}

Twelve studies were published in Chinese (Zhang et al., 2003; Yu et al., 2005; Wang and Li, 2007; Luo et al., 2008; Liu et al., 2009; Luo et al., 2010; He and Li, 2011; Wang, 2012; Zhou et al., 2012; Liu et al., 2014; Tian et al., 2015; Xiao et al., 2016) and three studies in English (Zhang et al., 2006; Chen et al., 2011; Gui et al., 2015) between 2006 and 2015. Fourteen studies used male BALB/C mice (Zhang et al., 2003; Yu et al., 2005; Zhang et al., 2006; Wang and Li, 2007; Luo et al., 2008; Liu et al., 2009; Luo et al., 2010; Chen et al., 2011; He and Li, 2011; Wang, 2012; Zhou et al., 2012; Liu et al., 2014; Gui et al., 2015; Tian et al., 2015) and one used male Kunming mice (Xiao et al., 2016). Eight studies used mice weighing 12-16 g for experiments (Zhang et al., 2003; Yu et al., 2005; Luo et al., 2008; Liu et al., 2009; Luo et al., 2010; He and Li, 2011; Liu et al., 2014; Tian et al., 2015), one used mice weighing 12-14 $\mathrm{g}$ (Wang and Li, 2007), three used mice weighing 14-16 g (Zhang et al., 2006; Wang, 2012; Zhou et al., 2012), one used mice weighing 18-22 g (Xiao et al., 2016) and two did not provide information on mouse weight (Chen et al., 2011; Gui et al., 2015). VM models were established by intraperitoneal injection of CVB3 in all studies. In terms of AS-IV dosage, one study utilized $4.36 \mathrm{~g} / \mathrm{kg} / \mathrm{d}$ (Xiao et al., 2016), three utilized $600 \mathrm{mg} / \mathrm{kg} / \mathrm{d}$ (Luo et al., 2008; Wang, 2012; Tian et al., 2015), one utilized $300 \mathrm{mg} / \mathrm{kg} / \mathrm{d}$ (Chen et al., 2011), one utilized 120

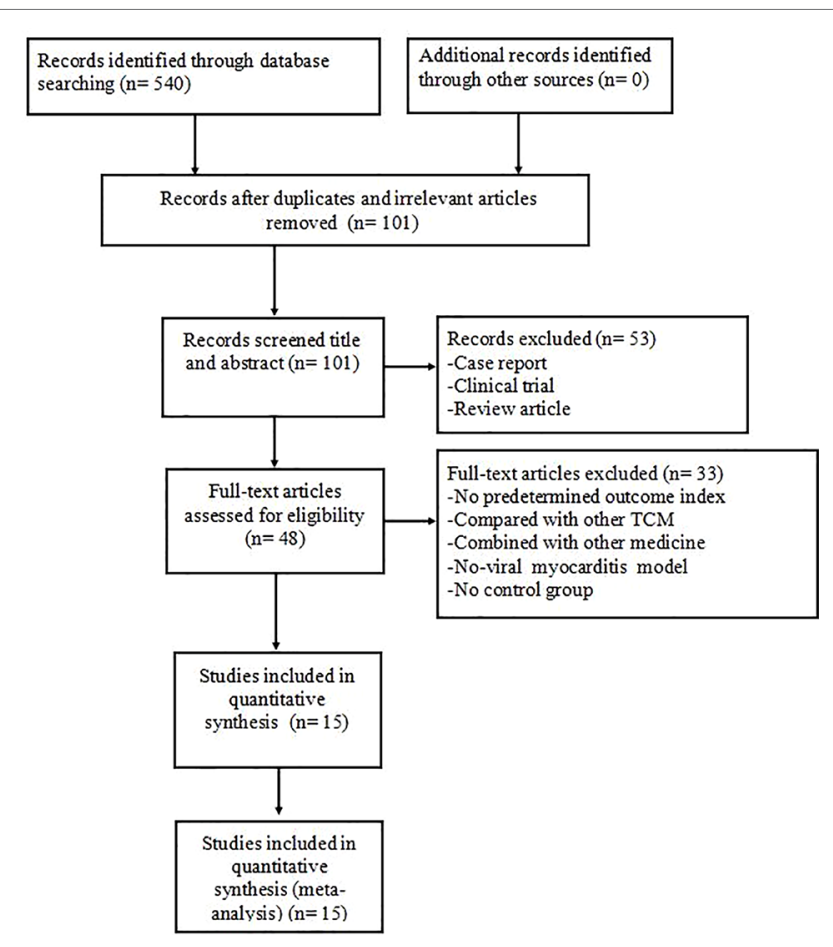

FIGURE 1 | Summary of the process for identifying candidate studies. $\mathrm{mg} / \mathrm{kg} / \mathrm{d}$ (Zhang et al., 2006), one utilized $40 \mathrm{mg} / \mathrm{kg} / \mathrm{d}$ (Gui et al., 2015), one utilized $1 \mathrm{mg} / \mathrm{kg} / \mathrm{d}$ (Wang and Li, 2007), two utilized $0.6 \mathrm{mg} / \mathrm{kg} / \mathrm{d}$ (Luo et al., 2010; He and Li, 2011), and five did not specify the dose used (Zhang et al., 2003; Yu et al., 2005; Liu et al., 2009; Zhou et al., 2012; Liu et al., 2014). Twelve studies utilized mortality rate as outcome measure (Zhang et al., 2003; Yu et al., 2005; Zhang et al., 2006; Wang and Li, 2007; Luo et al., 2008; Liu et al., 2009; Chen et al., 2011; He and Li, 2011; Wang, 2012; Liu et al., 2014; Gui et al., 2015; Tian et al., 2015) and histopathological changes of myocardium were assessed in nine studies (Yu et al., 2005; Wang and Li, 2007; Luo et al., 2008; Liu et al., 2009; Wang, 2012; Zhou et al., 2012; Liu et al., 2014; Gui et al., 2015; Tian et al., 2015). Left ventricular ejection fraction (LVEF) was reported in one study (Chen et al., 2011), shortening fraction (FS) in two studies (Chen et al., 2011; Gui et al., 2015) and left ventricular endsystolic dimensions (LVEDd) in two studies (Chen et al., 2011; Gui et al., 2015), but the level of ST-segment depression was not mentioned. The cardiac troponin I (cTnI) level was reported in one study (Gui et al., 2015), creatine kinase (CK) in one study (Gui et al., 2015), superoxide dismutase (SOD) in two studies (Wang and Li, 2007; Luo et al., 2008), glutathione (GSH) in two studies (Wang and Li, 2007; Luo et al., 2008), reative oxygen species (ROS) in one study (Wang and Li, 2007), tumor necrosis factor- $\alpha$ (TNF- $\alpha$ ) in two studies (Zhou et al., 2012; Gui et al., 2015), myocardial apoptosis index in two studies (Zhang et al., 2003; Luo et al., 2010), B-cell lymphoma-2 (Bcl-2) in two study (Luo et al., 2010), Bcl2-associated X (Bax) in one study (Luo et al., 2010), and nuclear factor $\kappa \mathrm{B}(\mathrm{NF}-\kappa \mathrm{B})$ in one study (Gui et al., 2015). The characteristics of the included studies are summarized in Table 1.

\section{Study Quality}

The quality of all the included studies was evaluated and scored from 2 to 5 . Two studies employed methods of random allocation (Zhang et al., 2006; Liu et al., 2014). Four studies specified the time of model induction before random allocation (Zhang et al., 2003; Wang and Li, 2007; Chen et al., 2011; Gui et al., 2015). Allocation to different groups was not concealed in any of the studies. Two studies declared compliance with animal welfare regulations (Zhang et al., 2006; Gui et al., 2015). However, no studies reported blinding of caregivers and the methods of random outcome assessment. One study described blinding of outcome assessor (Wang, 2012). All studies specified complete outcome data and no other sources of bias. Nine studies described the free of selective outcome reporting (Zhang et al., 2003; Yu et al., 2005; Zhang et al., 2006; Liu et al., 2009; Chen et al., 2011; Liu et al., 2014; Gui et al., 2015; Tian et al., 2015; Xiao et al., 2016). The methodological quality is summarized in Table 2.

\section{Effectiveness Mortality}

Meta-analysis of 12 studies (Zhang et al., 2003; Yu et al., 2005; Zhang et al., 2006; Wang and Li, 2007; Luo et al., 2008; Liu et al., 
TABLE 1 | Characteristics of the 15 included studies.

\begin{tabular}{|c|c|c|c|c|c|c|c|}
\hline Study (years) & $\begin{array}{l}\text { Species (Sex; } \\
\mathrm{n}=\text { experimental/ } \\
\text { control group) }\end{array}$ & Weight & Model (method) & $\begin{array}{l}\text { Treatment group } \\
\text { (Method to astragal } \\
\text { sides) }\end{array}$ & Control group & Outcome Index (time) & $\begin{array}{l}\text { Intergroup } \\
\text { Differences }\end{array}$ \\
\hline $\begin{array}{l}\text { Zhang et al. } \\
\text { (2003) }\end{array}$ & $\begin{array}{l}\text { BALB/C mice (male; } \\
\text { 18/18) }\end{array}$ & $12-16 \mathrm{~g}$ & $\begin{array}{l}\text { By intraperitoneal injection } \\
\text { of EMEM solution containing } \\
1 \times 10^{\wedge 9} \text { TCID50 CVB3 }(0.1 \mathrm{ml}) \\
\text { at the age of } 4 \text { weeks }\end{array}$ & $\begin{array}{l}\text { By intragastric } \\
\text { administration of } 9 \% \\
\text { AS-IV (0.1ml; qd) for } 7 d \\
\text { after establishing model }\end{array}$ & $\begin{array}{l}\text { By intragastric administration of } \\
\text { normal saline }(0.1 \mathrm{ml} \text {; qd) for } 7 \mathrm{~d} \\
\text { after establishing model }\end{array}$ & $\begin{array}{l}\text { 1.Mortality rate } \\
\text { 2.CVF } \\
\text { 3.PIP } \\
\text { 4. PIIINP } \\
\text { 5.Myocardial apoptosis index }\end{array}$ & $\begin{array}{l}1 . P<0.05 \\
2 . P<0.01 \\
3 . P<0.01 \\
4 . P<0.01 \\
5 . P<0.01\end{array}$ \\
\hline Yu et al. (2005) & $\begin{array}{l}\text { BALB/C mice (male; } \\
\text { 20/20) }\end{array}$ & $12-16 \mathrm{~g}$ & $\begin{array}{l}\text { By intraperitoneal injection of } \\
\text { CVB3 at the age of } 4 \text { weeks }\end{array}$ & $\begin{array}{l}\text { By intragastric } \\
\text { administration of } 9 \% \\
\text { AS-IV (0.1ml; qd) for } 7 d \\
\text { after establishing model }\end{array}$ & $\begin{array}{l}\text { By intragastric administration of } \\
\text { sodium carboxymethyl cellulose } \\
\text { solution ( } 0.1 \mathrm{ml} \text {; qd) for } 7 \mathrm{~d} \text { after } \\
\text { establishing model }\end{array}$ & $\begin{array}{l}\text { 1. Mortality rate } \\
\text { 2.Histopathological changes of } \\
\text { myocardium }\end{array}$ & $\begin{array}{l}1 . P<0.05 \\
2 . P<0.01\end{array}$ \\
\hline $\begin{array}{l}\text { Zhang et al. } \\
\text { (2006) }\end{array}$ & $\begin{array}{l}\text { BALB/C Mice (male; } \\
\text { 15/16) }\end{array}$ & $14-16 \mathrm{~g}$ & $\begin{array}{l}\text { By intraperitoneal injection of } \\
\text { CVB3 at the age of } 4 \text { weeks }\end{array}$ & $\begin{array}{l}\text { By intragastric } \\
\text { administration of AS-IV } \\
\text { (120mg/kg; qd) for } 7 d \\
\text { after establishing model }\end{array}$ & $\begin{array}{l}\text { By intragastric administration of } \\
\text { isometric normal saline solution } \\
\text { (qd) for } 7 \mathrm{~d} \text { after establishing } \\
\text { model }\end{array}$ & $\begin{array}{l}\text { 1.Mortality rate } \\
\text { 2.HW/BW ratios } \\
\text { 3.Virus titers of hearts } \\
\text { 4.Scores of necrosis and } \\
\text { infiltration }\end{array}$ & $\begin{array}{l}1 . P<0.01 \\
2 . P<0.01 \\
\text { 3.P }<0.01 \\
\text { 4.P }<0.01\end{array}$ \\
\hline $\begin{array}{l}\text { Wang and Li } \\
\text { (2007) }\end{array}$ & $\begin{array}{l}\text { BALB/C Mice (male; } \\
\text { 30/30) }\end{array}$ & $12-14 \mathrm{~g}$ & $\begin{array}{l}\text { By intraperitoneal injection of } \\
\text { RPIM- } 1640 \text { solution containing } \\
1 \times 10^{\wedge 5} \text { TCID50 CVB3 at the } \\
\text { age of } 4 \text { weeks }\end{array}$ & $\begin{array}{l}\text { By intragastric } \\
\text { administration of AS-IV } \\
(1 \mathrm{mg} / \mathrm{kg} ; \mathrm{qd}) \text { for } 7 \mathrm{~d} \text { after } \\
\text { establishing model }\end{array}$ & $\begin{array}{l}\text { By intragastric administration of } \\
\text { isometric sodium carboxymethyl } \\
\text { cellulose solution (qd) for } 7 d \\
\text { after establishing model }\end{array}$ & $\begin{array}{l}\text { 1.Mortality rate } \\
\text { 2. Histopathological changes of } \\
\text { myocardium } \\
\text { 3.T-SOD } \\
\text { 4.GSH-PX } \\
\text { 5.CAT } \\
\text { 6.MPO } \\
\text { 7.ROS }\end{array}$ & $\begin{array}{l}1 . P<0.01 \\
2 . P<0.05 \\
3 . P<0.01 \\
4 . P<0.01 \\
5 . P<0.01 \\
6 . P<0.01 \\
7 . P<0.01\end{array}$ \\
\hline Luo et al. (2008) & $\begin{array}{l}\text { BALB/C mice (male; } \\
\text { 10/10) }\end{array}$ & $12-16 \mathrm{~g}$ & $\begin{array}{l}\text { By intraperitoneal injection } \\
\text { of EMEM solution containing } \\
1 \times 10^{\wedge} 2 \mathrm{TCID} 50 \text { CVB3 }(0.1 \mathrm{ml}) \\
\text { at the age of } 4 \text { weeks }\end{array}$ & $\begin{array}{l}\text { By intragastric } \\
\text { administration of } 9 \% \\
\text { AS-IV (600mg/kg) } \\
(0.1 \mathrm{ml} \text {; qd) for } 7 \mathrm{~d} \text { after } \\
\text { establishing model }\end{array}$ & $\begin{array}{l}\text { By intragastric administration of } \\
\text { normal saline }(0.1 \mathrm{ml} \text {; qd) for } 7 \mathrm{~d} \\
\text { after establishing model }\end{array}$ & $\begin{array}{l}\text { 1.Mortality rate } \\
\text { 2.Histopathological changes of } \\
\text { myocardium } \\
\text { 3.T-SOD } \\
\text { 4.GSH-PX } \\
\text { 5.CAT }\end{array}$ & $\begin{array}{l}1 . P<0.05 \\
2 . P<0.01 \\
3 . P<0.05 \\
4 . P<0.05 \\
5 . P<0.05\end{array}$ \\
\hline Liu et al. (2009) & $\begin{array}{l}\text { BALB/C mice (male; } \\
15 / 15)\end{array}$ & $12-16 \mathrm{~g}$ & $\begin{array}{l}\text { By intraperitoneal injection of } \\
\text { virus culture medium containing } \\
1 \times 10^{\wedge} 2 \text { TCID50 CVB3 }(0.1 \mathrm{ml}) \\
\text { at the age of } 4 \text { weeks }\end{array}$ & $\begin{array}{l}\text { By intragastric } \\
\text { administration of } 9 \% \\
\text { AS-IV (0.1ml; qd) for } 14 \mathrm{~d} \\
\text { after establishing model }\end{array}$ & $\begin{array}{l}\text { By intragastric administration of } \\
\text { sodium carboxymethyl cellulose } \\
\text { solution ( } 0.1 \mathrm{ml} \text {; qd) for } 14 \mathrm{~d} \text { after } \\
\text { establishing model }\end{array}$ & $\begin{array}{l}\text { 1.Mortality rate } \\
\text { 2.Histopathological changes of } \\
\text { myocardium } \\
\text { 3.MIF protein }\end{array}$ & $\begin{array}{l}1 . P<0.05 \\
2 . P<0.01 \\
3 . P<0.01\end{array}$ \\
\hline Luo et al. (2010) & $\begin{array}{l}\text { BALB/C mice (male; } \\
\text { 10/10) }\end{array}$ & $12-16 \mathrm{~g}$ & $\begin{array}{l}\text { By intraperitoneal injection of } \\
\text { EMEM solution containing } 1 \\
\times 10^{\wedge 5} \text { TCID } 50 \text { CVB3 }(0.1 \mathrm{ml}) \text { at } \\
\text { the age of } 4 \text { weeks }\end{array}$ & $\begin{array}{l}\text { By intragastric } \\
\text { administration of } 9 \% \\
\text { AS-IV (0.6mg } / \mathrm{kg}) \\
(0.1 \mathrm{ml} \text {; qd) for } 7 \mathrm{~d} \text { after } \\
\text { establishing model }\end{array}$ & $\begin{array}{l}\text { By intragastric administration of } \\
\text { normal saline }(0.1 \mathrm{ml} \text {; qd) for } 7 \mathrm{~d} \\
\text { after establishing model }\end{array}$ & $\begin{array}{l}\text { 1.Myocardial apoptosis index } \\
\text { 2.Bcl-2 } \\
\text { 3.Bax }\end{array}$ & $\begin{array}{l}1 . P<0.01 \\
\text { 2.P }<0.01 \\
\text { 3.P }<0.01\end{array}$ \\
\hline Chen et al. (2011) & $\begin{array}{l}\text { BALB/C mice (male; } \\
\text { 20/30) }\end{array}$ & NM & $\begin{array}{l}\text { By intraperitoneal injection of } \\
\text { CVB3 (100ul) at the age of } 4 \\
\text { weeks and treatments monthly } \\
\text { for } 9 \text { month to induce dilated } \\
\text { cardiomyopathy }\end{array}$ & $\begin{array}{l}\text { By intragastric } \\
\text { administration of AS-IV } \\
\text { ( } 300 \mathrm{mg} / \mathrm{L} ; \mathrm{qd} \text { ) containing } \\
\text { drinking water after } \\
\text { establishing model }\end{array}$ & $\begin{array}{l}\text { By intragastric administration of } \\
\text { isometric plain drinking water } \\
\text { (qd) after establishing model }\end{array}$ & $\begin{array}{l}\text { 1.Mortality rate } \\
\text { 2.LVEDd } \\
\text { 3.LVEDs } \\
\text { 4.LVEF } \\
\text { 5.FS } \\
\text { 6.PICP } \\
\text { 7.PINP } \\
\text { 8.PICP/PINP ratio } \\
\text { 9.TGF- } \beta 1 \\
\text { 10.pSmad2/3 } \\
\text { 11.Smad4 } \\
\text { 12.Smad7 }\end{array}$ & $\begin{array}{l}1 . P<0.05 \\
2 . P<0.001 \\
3 . P<0.001 \\
4 . P<0.001 \\
5 . P<0.001 \\
6 . P<0.05 \\
7 . P>0.05 \\
8 . P<0.01 \\
9 . P<0.05 \\
10 . P<0.05 \\
11 . P<0.01 \\
12 . P>0.05\end{array}$ \\
\hline $\mathrm{He}$ and Li (2011) & $\begin{array}{l}\text { BALB/C mice (male; } \\
\text { 15/15) }\end{array}$ & $12-16 \mathrm{~g}$ & $\begin{array}{l}\text { By intraperitoneal injection } \\
\text { of Eagle' s culture media } \\
\text { containing } 1 \times 10^{\wedge 2} \text { TCID50 } \\
\text { CVB3 }(0.1 \mathrm{ml}) \text { at the age of } \\
4 \text { weeks }\end{array}$ & $\begin{array}{l}\text { By intragastric } \\
\text { administration of } 9 \% \\
\text { AS-IV }(0.6 \mathrm{mg} / \mathrm{kg} \text {; qd) for } \\
15 \mathrm{~d} \text { after establishing } \\
\text { model }\end{array}$ & $\begin{array}{l}\text { By intragastric administration } \\
\text { of isometric hydroxymethyl } \\
\text { cellulose sodium solution (qd) } \\
\text { for } 15 \mathrm{~d} \text { after establishing model }\end{array}$ & $\begin{array}{l}\text { 1.IGF-1 in plasma } \\
\text { 2.IGF-1 Protein in myocardium } \\
\text { 3.IGF-1R Protein in } \\
\text { myocardium } \\
\text { 4.IGFBP3 Protein in } \\
\text { myocardium }\end{array}$ & $\begin{array}{l}1 . P<0.05 \\
2 . P<0.05 \\
3 . P<0.05 \\
4 . P<0.05\end{array}$ \\
\hline
\end{tabular}


TABLE 1 | Continued

\begin{tabular}{|c|c|c|c|c|c|c|c|}
\hline Study (years) & $\begin{array}{l}\text { Species (Sex; } \\
\mathrm{n}=\text { experimental/ } \\
\text { control group) }\end{array}$ & Weight & Model (method) & $\begin{array}{l}\text { Treatment group } \\
\text { (Method to astragal } \\
\text { sides) }\end{array}$ & Control group & Outcome Index (time) & $\begin{array}{l}\text { Intergroup } \\
\text { Differences }\end{array}$ \\
\hline Wang (2012) & $\begin{array}{l}\text { BALB/C mice } \\
\text { (male; 30/30) }\end{array}$ & $14-16 \mathrm{~g}$ & $\begin{array}{l}\text { By intraperitoneal injection } \\
\text { of CVB3 }(0.1 \mathrm{ml}) \text { at the age } \\
\text { of } 4 \text { weeks }\end{array}$ & $\begin{array}{l}\text { By intragastric } \\
\text { administration of } \\
\text { AS-IV }(0.1 \mathrm{ml} \text {; qd) for } \\
7 \mathrm{~d} \text { after establishing } \\
\text { model }\end{array}$ & $\begin{array}{l}\text { By intragastric } \\
\text { administration of normal } \\
\text { saline }(0.1 \mathrm{ml} \text {; qd) for } 7 \mathrm{~d} \\
\text { after establishing model }\end{array}$ & $\begin{array}{l}\text { 1. Histopathological } \\
\text { changes of myocardium } \\
\text { 2.P38MAPK }\end{array}$ & $\begin{array}{l}1 . P<0.05 \\
2 . P<0.05\end{array}$ \\
\hline $\begin{array}{l}\text { Zhou et al. } \\
\text { (2012) }\end{array}$ & $\begin{array}{l}\text { BALB/C mice } \\
\text { (male; 30/30) }\end{array}$ & $14-16 g$ & $\begin{array}{l}\text { By intraperitoneal injection } \\
\text { of } 1 \times 10^{\wedge} 2 \text { TCID50 CVB3 } \\
(0.1 \mathrm{ml}) \text { at the age of } 4 \\
\text { weeks }\end{array}$ & $\begin{array}{l}\text { By intragastric } \\
\text { administration of } \\
\text { AS-IV (0.1ml; qd) for } \\
7 \mathrm{~d} \text { after establishing } \\
\text { model }\end{array}$ & $\begin{array}{l}\text { By intragastric } \\
\text { administration of normal } \\
\text { saline }(0.1 \mathrm{ml} \text {; qd) for } 7 \mathrm{~d} \\
\text { after establishing model }\end{array}$ & $\begin{array}{l}\text { 1. Histopathological } \\
\text { changes of myocardium } \\
\text { 2.TNF- } \alpha\end{array}$ & $\begin{array}{l}1 . P<0.05 \\
2 . P<0.05\end{array}$ \\
\hline Liu et al. (2014) & $\begin{array}{l}\text { BALB/C mice } \\
\text { (male; 20/20) }\end{array}$ & $12-16 g$ & $\begin{array}{l}\text { By intraperitoneal injection } \\
\text { of virus culture medium } \\
\text { containing } 1 \times 10^{\wedge} 2 \\
\text { TCID50 CVB3 }(0.1 \mathrm{ml}) \text { at } \\
\text { the age of } 4 \text { weeks }\end{array}$ & $\begin{array}{l}\text { By intragastric } \\
\text { administration of } 9 \% \\
\text { AS-IV }(0.1 \mathrm{ml} \text {; qd) for } \\
\text { 14d after establishing } \\
\text { model }\end{array}$ & $\begin{array}{l}\text { By intragastric } \\
\text { administration of sodium } \\
\text { carboxymethyl cellulose } \\
\text { solution }(0.1 \mathrm{ml} \text {; qd) for } 14 \mathrm{~d} \\
\text { after establishing model }\end{array}$ & $\begin{array}{l}\text { 1. Mortality rate } \\
\text { 2. Histopathological } \\
\text { changes of myocardium } \\
\text { 3.Th17 } \\
\text { 4.IL-23 } \\
\text { 5.IL-17 }\end{array}$ & $\begin{array}{l}1 . P>0.003 \\
2 . P<0.05 \\
\text { 3.P }<0.05 \\
4 . P<0.05 \\
5 . P<0.05\end{array}$ \\
\hline $\begin{array}{l}\text { Gui et al. } \\
(2015)\end{array}$ & $\begin{array}{l}\text { BALB/C mice } \\
\text { (male; 15/15) }\end{array}$ & NM & $\begin{array}{l}\text { By intraperitoneal injection } \\
\text { of } 1 \times 10^{\wedge}{ }^{3} \text { TCID } 50 \text { CVB3 } \\
\text { at the age of } 6 \text { weeks }\end{array}$ & $\begin{array}{l}\text { By intragastric } \\
\text { injection of AS-IV } \\
(40 \mathrm{mg} / \mathrm{kg} ; \mathrm{gd}) \text { for } \\
7 \mathrm{~d} \text { after establishing } \\
\text { model }\end{array}$ & $\begin{array}{l}\text { By intragastric injection of } \\
\text { isometric normal saline ( } q d \text { ) } \\
\text { for } 7 d \text { after establishing } \\
\text { model }\end{array}$ & $\begin{array}{l}\text { 1.The changes of } \\
\text { body weight } \\
\text { 2.Mortality rate } \\
\text { 3.CK-MB } \\
\text { 4.cTnl } \\
\text { 5.Myocarditis score } \\
\text { 6. PWd } \\
\text { 7.SWd } \\
\text { 8.LVEDd } \\
\text { 9.LVEDs } \\
\text { 10.EF } \\
\text { 11.FS } \\
\text { 12.HR } \\
\text { 13.TNF- } \alpha \text { in myocardium } \\
\text { 14.IL-1 } \beta \text { in myocardium } \\
\text { 15.IL-6 in myocardium } \\
\text { 16.MCP-1 in myocardium } \\
\text { 17.TNF- } \alpha \text { in serum } \\
\text { 18.IL-1 } \beta \text { in serum } \\
\text { 19.IL-6 in serum } \\
\text { 20.MCP-1 in serum } \\
\text { 21.CD3 } \\
\text { 22.CD11b } \\
\text { 23.NF- } \kappa B \text { DNA } \\
\text { 24.A20 }\end{array}$ & $\begin{array}{l}\text { 1.P }<0.01 \\
\text { 2.P }<0.01 \\
\text { 3.P }<0.01 \\
\text { 4.P }<0.01 \\
\text { 5.P }<0.01 \\
\text { 6.P }<0.01 \\
\text { 7.P }>0.05 \\
\text { 8.P }<0.01 \\
\text { 9.P }<0.01 \\
\text { 10.P }<0.01 \\
\text { 11.P }<0.01 \\
\text { 12.P }>0.05 \\
\text { 13.P }<0.001 \\
\text { 14.P }<0.01 \\
\text { 15.P }<0.001 \\
\text { 16.P }<0.001 \\
\text { 17.P }<0.001 \\
\text { 18.P }<0.001 \\
\text { 19.P }<0.001 \\
\text { 20.P }<0.001 \\
\text { 21.P }<0.001 \\
\text { 22.P }<0.01 \\
\text { 23.P }<0.01 \\
\text { 24.P }<0.001\end{array}$ \\
\hline $\begin{array}{l}\text { Tian et al. } \\
(2015)\end{array}$ & $\begin{array}{l}\text { BALB/C mice } \\
\text { (male; 20/20) }\end{array}$ & $12-16 \mathrm{~g}$ & $\begin{array}{l}\text { By intraperitoneal injection } \\
\text { of Eagle' s culture media } \\
\text { containing } 1 \times 10^{\wedge 2} \\
\text { TCID50 CVB3 }(0.1 \mathrm{ml}) \text { at } \\
\text { the age of } 4 \text { weeks }\end{array}$ & $\begin{array}{l}\text { By intragastric } \\
\text { administration of } 9 \% \\
\text { AS-IV (600mg/kg; } \\
0.1 \mathrm{ml} \text {; qd) for } 7 \mathrm{~d} \text { after } \\
\text { establishing model }\end{array}$ & $\begin{array}{l}\text { By intragastric } \\
\text { administration of sodium } \\
\text { carboxymethyl cellulose } \\
\text { solution ( } 0.1 \mathrm{ml} \text {; qd) for } 7 d \\
\text { after establishing model }\end{array}$ & $\begin{array}{l}\text { 1.Mortality rate } \\
\text { 2.Histopathological } \\
\text { changes of myocardium } \\
\text { 3.TL1A protein }\end{array}$ & $\begin{array}{l}1 . P<0.05 \\
2 . P<0.01 \\
3 . P<0.01\end{array}$ \\
\hline $\begin{array}{l}\text { Xiao et al. } \\
(2016)\end{array}$ & $\begin{array}{l}\text { Kunming mice } \\
\text { (male; 15/15) }\end{array}$ & $18-22 \mathrm{~g}$ & $\begin{array}{l}\text { By intraperitoneal injection } \\
\text { of CVB3 }(0.1 \mathrm{ml} \text {; qod) for } \\
4 \text { times }\end{array}$ & $\begin{array}{l}\text { By intragastric } \\
\text { administration of } \\
\text { AS-IV }(4.36 \mathrm{~g} / \mathrm{kg} \text {; } \\
\text { qd) for } 15 \mathrm{~d} \text { after } \\
\text { establishing model }\end{array}$ & $\begin{array}{l}\text { By intragastric administration } \\
\text { of isometric } 0.5 \% \text { sodium } \\
\text { carboxymethyl cellulose } \\
\text { solution ( } 20 \mathrm{ml} / \mathrm{kg} \text {; qd) for } \\
15 \mathrm{~d} \text { after establishing model }\end{array}$ & $\begin{array}{l}\text { 1.TIMP-1 } \\
\text { 2.MMP-1 }\end{array}$ & $\begin{array}{l}1 . P<0.01 \\
2 . P<0.01\end{array}$ \\
\hline
\end{tabular}

A20, TNF- $\alpha$ induced protein 3; AS-IV, astragaloside IV; Bax, Bcl2-associated X; Bcl-2, B-cell lymphoma-2; CAT, catalase; cTnl, cardiac troponin I; CD3, clusters of differentiation 3; CD11b, clusters of differentiation 11b; CVB3, coxsackievirus B3; CVF, collagen volume fraction; EF, ejection fraction; EMEM, Eagle's minimal essential medium; FS, shortening fraction; GSH-PX, glutathion peroxidase; HR, heart rate; HW/BW, heart weight/body weight; IGF-1, insulin-like growth factor-1; IGF-1R, insulin-like growth factor-1R; IGFBP3, Insulin-

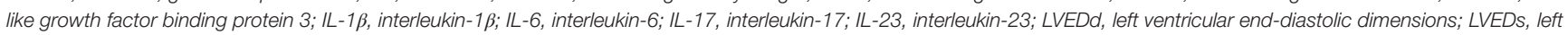
ventricular endsystolic dimensions; LVEF, left ventricular ejection fraction; MCP-1, monocyte chemoattractant protein-1; MIF, macrophage migration inhibitory factor; MMP-1, matrix metalloproteinase-1; MPO, myeloperoxidase; NF- $\kappa$, nuclear factor- $\kappa B ;$ P38MAPK, P38 mitogen-activated protein kinase; PICP, carboxyterminal propeptide of type I procollagen; PINP, aminoterminal propeptide of type I procollagen; PIP, propeptide of procollagen type I; PIIINP, procollagen type III aminoterminal peptide; pSmad2/3, phosphorylated small mothers against decapentaplegic 2/3; PWd, posterior wall thickness in end diastole; RPIM-1640, Roswell Park Memorials Institute 1640; ROS, reative oxygen species; Smad4, drosophila mothers against decapentaplegic 4; Smad7, drosophila mothers against decapentaplegic7; SWd, septal wall thickness; TCID50, tissue culture infectious dose 50; TGF$\beta 1$, transforming growth factor- $\beta 1$; Th17, helper T cell 17; TIMP-1, tissue inhibitor of metalloproteinase-1; TL1A, TNF-like ligand 1 aberrance; TNF- $\alpha$, tumor necrosis factor- $\alpha$; T-SOD, total superoxide dimutase. 
TABLE 2 | Quality assessment of included studies.

\begin{tabular}{|c|c|c|c|c|c|c|c|c|c|c|c|}
\hline Study & A & B & C & D & $\mathbf{E}$ & $\mathbf{F}$ & G & $\mathbf{H}$ & $\mathbf{I}$ & $\mathbf{J}$ & Total \\
\hline Zhang et al. (2003) & $?$ & + & - & - & - & - & - & + & + & + & 4 \\
\hline Yu et al. (2005) & - & - & - & - & - & - & - & + & + & + & 3 \\
\hline Zhang et al. (2006) & + & - & - & + & - & - & - & + & + & + & 5 \\
\hline Wang and Li (2007) & $?$ & + & - & - & - & - & - & + & $?$ & + & 3 \\
\hline Luo et al. (2008) & $?$ & - & - & - & - & - & - & + & $?$ & + & 2 \\
\hline Liu et al. (2009) & $?$ & - & - & - & - & - & - & + & + & + & 3 \\
\hline Luo et al. (2010) & $?$ & - & - & - & - & - & - & + & $?$ & + & 2 \\
\hline Chen et al. (2011) & $?$ & + & - & - & - & - & - & + & + & + & 4 \\
\hline He and Li (2011) & $?$ & - & - & - & - & - & - & + & $?$ & + & 2 \\
\hline Wang (2012) & $?$ & - & - & - & - & $?$ & + & + & $?$ & + & 3 \\
\hline Zhou et al. (2012) & $?$ & - & - & - & - & - & - & + & $?$ & + & 2 \\
\hline Liu et al. (2014) & + & - & - & - & - & - & - & + & + & + & 4 \\
\hline Gui et al. (2015) & - & + & - & + & - & - & - & + & + & + & 5 \\
\hline Tian et al. (2015) & $?$ & - & - & - & - & - & - & + & + & + & 3 \\
\hline Xiao et al. (2016) & $?$ & - & - & - & - & - & - & + & + & + & 3 \\
\hline
\end{tabular}

sequence generation; B: baseline characteristics; C: allocation concealment; D: random housing and animal welfare; E: blinding of caregivers and/or investigators; F: random outcome assessment; G: blinding of outcome assessor; H: complete outcome data; I: selective outcome reporting; J: other sources of bias. "+" indicates low risk of bias; "-" indicates high risk of bias; and "?" indicates an unclear risk of bias.

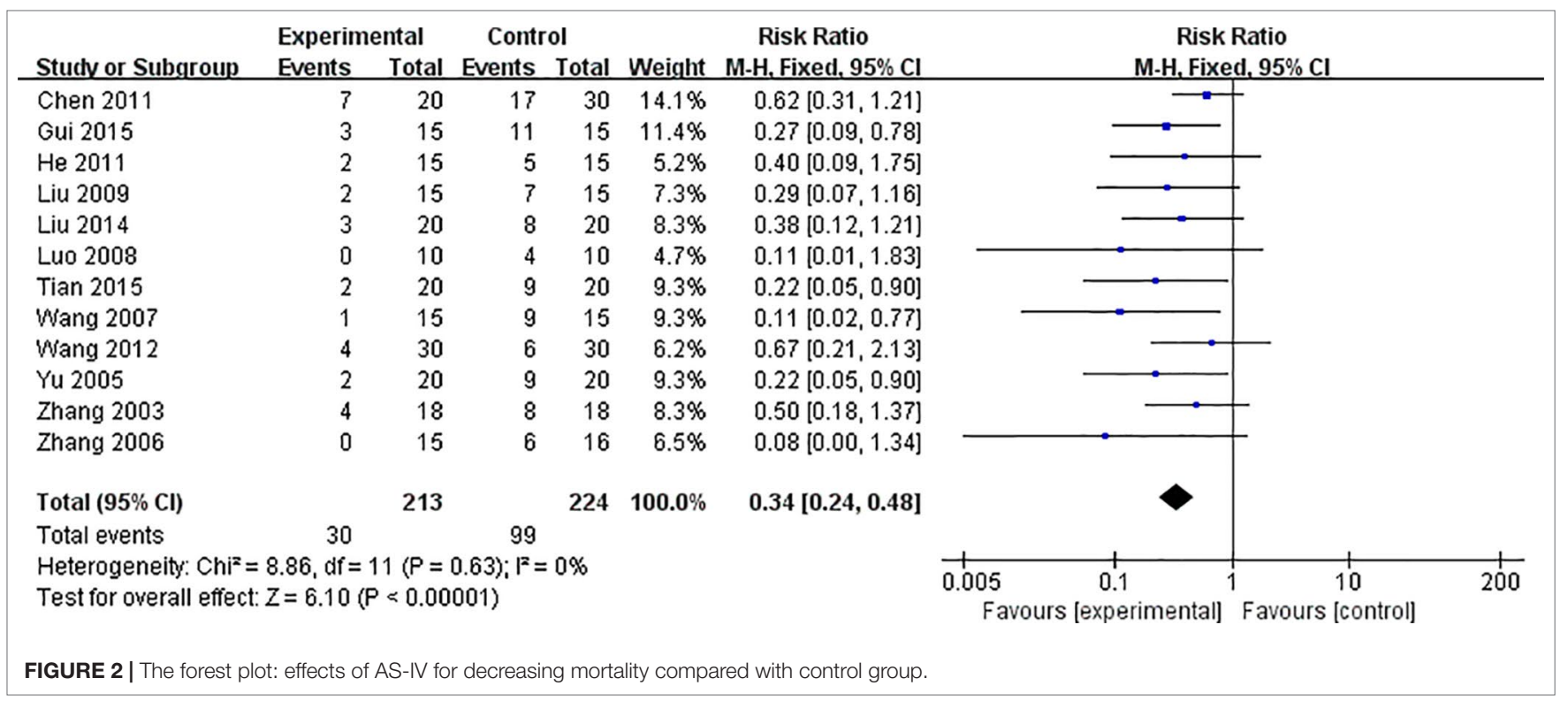

\begin{tabular}{|c|c|c|c|c|c|c|c|c|c|c|c|}
\hline \multirow[b]{2}{*}{ Study or Subgroup } & \multicolumn{3}{|c|}{ Experimental } & \multicolumn{2}{|c|}{ Control } & \multirow[b]{2}{*}{ Total } & \multirow[b]{2}{*}{ Weight } & \multirow{2}{*}{$\begin{array}{l}\text { Mean Difference } \\
\text { IV, Fixed, } 95 \% \mathrm{Cl}\end{array}$} & \multirow{2}{*}{\multicolumn{3}{|c|}{$\begin{array}{l}\text { Mean Difference } \\
\text { N, Fixed, } 95 \% \mathrm{Cl}\end{array}$}} \\
\hline & Mean & SD & Total & Mean & SD & & & & & & \\
\hline Gui 2015 & 1.27 & 0.1 & 12 & 3.69 & 0.16 & 4 & & Not estimable & & & \\
\hline Liu 2009 & $1.5 ?$ & 0.32 & 13 & 2.63 & 0.56 & 8 & $18.1 \%$ & $-1.06[-1.49,-0.63]$ & & & \\
\hline Liu 2014 & 1.68 & 0.25 & 17 & 2.67 & 0.48 & 12 & $37.3 \%$ & $-0.99[-1.29,-0.69]$ & -m- & & \\
\hline Luo 2008 & 1.86 & 0.59 & 10 & 3.23 & 0.83 & 6 & $5.7 \%$ & $-1.37[-2.13,-0.61]$ & \multirow[b]{2}{*}{$\longrightarrow$} & & \\
\hline Tian 2015 & 1.86 & 0.69 & 18 & 3.12 & 0.83 & 11 & $9.6 \%$ & $-1.26[-1.84,-0.68]$ & & & \\
\hline Wang $200 ?$ & 1.8 & 0.53 & 14 & 3.16 & 0.46 & 6 & $15.4 \%$ & $-1.36[-1.82,-0.90]$ & - & & \\
\hline Yu 2005 & 1.86 & 0.69 & 18 & 3.13 & 0.83 & 11 & $9.6 \%$ & $-1.27[-1.85,-0.69]$ & $\longrightarrow$ & & \\
\hline Zhou 2012 & 1.09 & 0.7 & 8 & 2.1 & 1.04 & 8 & $4.3 \%$ & $-1.01[-1.88,-0.14]$ & \multirow[b]{2}{*}{$\diamond$} & & \\
\hline \multirow{3}{*}{\multicolumn{6}{|c|}{$\begin{array}{l}\text { Total }(95 \% \mathrm{Cl}) \\
\text { Heterogeneity: } \mathrm{Chi}^{2}=2.78, \mathrm{df}=6(\mathrm{P}=0.84) ;\left.\right|^{2}=0 \% \\
\text { Test for overall effect: } Z=12.29(P<0.00001)\end{array}$}} & 62 & $100.0 \%$ & $-1.13[-1.32,-0.95]$ & & & \\
\hline & & & & & & & & & $\begin{array}{l}+ \\
-4\end{array}$ & 2 & + \\
\hline & & & & & & & & & Favours [experimental] & Favours [control] & \\
\hline
\end{tabular}




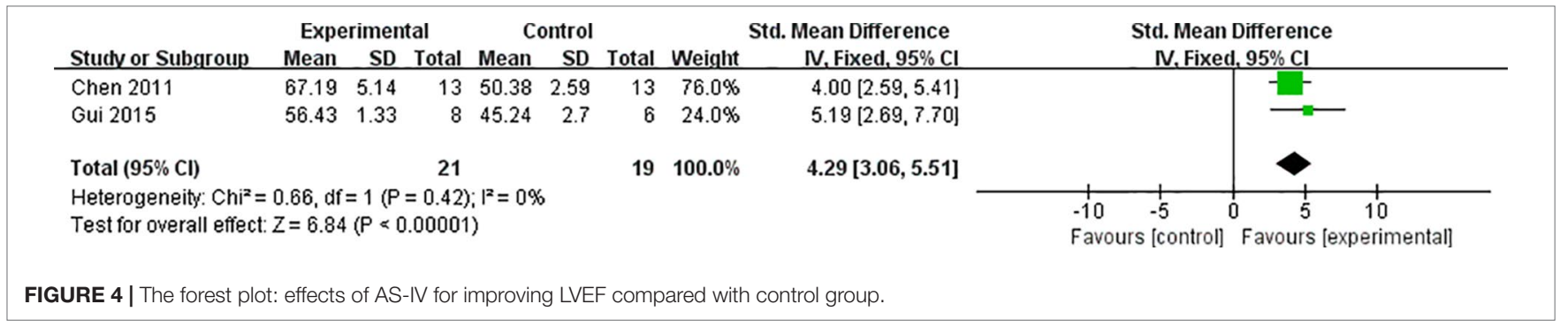

\begin{tabular}{|c|c|c|c|c|c|c|c|c|c|c|c|}
\hline \multirow{2}{*}{ Study or Subgroup } & \multicolumn{3}{|c|}{ Experimental } & \multicolumn{2}{|c|}{ Control } & \multicolumn{3}{|r|}{ Std. Mean Difference } & \multirow{2}{*}{\multicolumn{2}{|c|}{$\begin{array}{l}\text { Std. Mean Difference } \\
\text { IV, Fixed, } 95 \% \mathrm{Cl}\end{array}$}} & \\
\hline & Mean & SD & Total & Mean & SD & lotal & Weight & IV, Fixed, 95\% Cl & & & \\
\hline Gui 2015 & $\begin{array}{r}1.8 \\
2.148\end{array}$ & $\begin{array}{l}0.15 \\
0.02\end{array}$ & $\begin{array}{r}13 \\
8\end{array}$ & $\begin{array}{l}2.32 \\
2.76\end{array}$ & $\begin{array}{l}0.16 \\
0.376\end{array}$ & $\begin{array}{r}13 \\
6\end{array}$ & $40.9 \%$ & $\begin{array}{l}-3.23[-4.48,-2.02] \\
-2.36[-3.83,-0.88]\end{array}$ & $\rightarrow-$ & & \\
\hline Total $(95 \% \mathrm{Cl})$ & & & 21 & & & 19 & $100.0 \%$ & $-2.88[-3.83,-1.94]$ & & & \\
\hline \multicolumn{9}{|c|}{$\begin{array}{l}\text { Heterogeneity: } \mathrm{Chi}^{2}=0.83, \mathrm{df}=1(P=0.36) ; \mathrm{l}^{2}=0 \% \\
\text { Test for overall effect: } Z=5.98(P<0.00001)\end{array}$} & $\begin{array}{ccc}-10 & -5 & 0 \\
\text { Favours [experimental] }\end{array}$ & $\begin{array}{l}1 \\
0 \\
\text { Favours [control] }\end{array}$ & 10 \\
\hline
\end{tabular}

2009; Chen et al., 2011; He and Li, 2011; Wang, 2012; Liu et al., 2014; Gui et al., 2015; Tian et al., 2015) showed that AS-IV induces a significant reduction in mortality of VM animals, compared with control $[\mathrm{n}=437, \mathrm{RR} 0.34,95 \% \mathrm{CI}(0.24$ to 0.48$)$, $\mathrm{P}<0.0001$; heterogeneity: $\chi^{2}=8.86, \mathrm{I}^{2}=0 \%$ ] (Figure 2).

\section{Histopathological Changes of Myocardium}

Meta-analysis of eight studies (Yu et al., 2005; Wang and Li, 2007; Luo et al., 2008; Liu et al., 2009; Zhou et al., 2012; Liu et al., 2014; Gui et al., 2015; Tian et al., 2015) revealed significant effects of AS-IV on reducing inflammation of the myocardium and pathological score in VM animals, compared with control [ $\mathrm{n}=$ 176, MD -1.83 95\% CI ( -1.95 to -1.71$), \mathrm{P}<0.0001$; heterogeneity: $\left.\chi^{2}=107.56, I^{2}=93 \%\right]$. After sensitivity analyses, we removed one study (Gui et al., 2015) that modeling in mice at the age of 6 weeks. Meta-analysis of seven studies showed significant effects of AS-IV on reducing myocardial inflammation and pathological scores in VM animals [ $\mathrm{n}=160, \mathrm{MD}-1.1395 \% \mathrm{CI}(-1.32$ to -0.95$)$, $\mathrm{P}<0.0001$; heterogeneity: $\chi^{2}=2.78, \mathrm{I}^{2}=0 \%$ ] (Figure 3).

\section{LVEF}

Data from meta-analysis of two studies (Chen et al., 2011; Gui et al., 2015) showed that AS-IV treatment induced significant improvement of LVEF in animals with VM [n = 40 SMD 4.29 95\% CI (3.06 to 5.51), P < 0.0001; heterogeneity: $\chi^{2}=0.66, \mathrm{I}^{2}=$ 0\%] (Figure 4).

\section{LVEDs}

Data from meta-analysis of two studies (Chen et al., 2011; Gui et al., 2015) revealed significant suppressive effects of

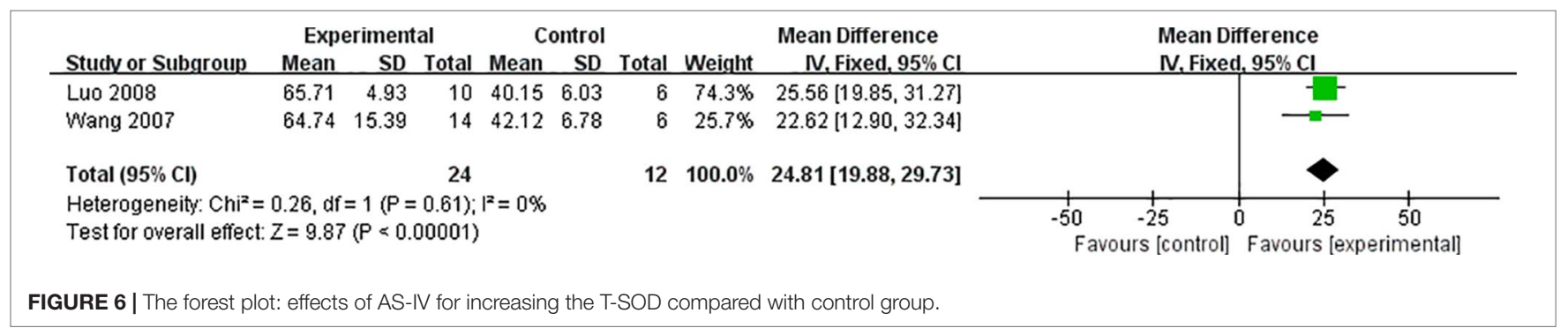

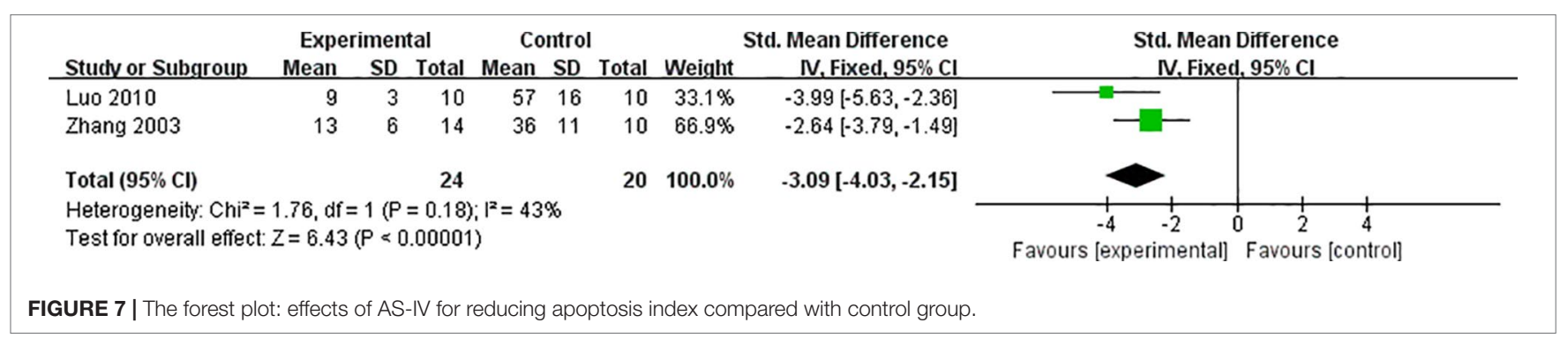




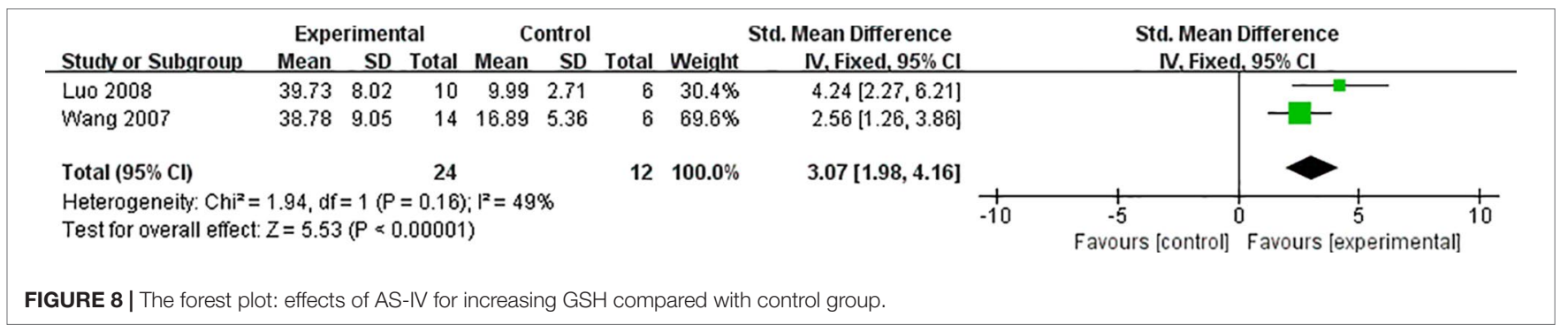

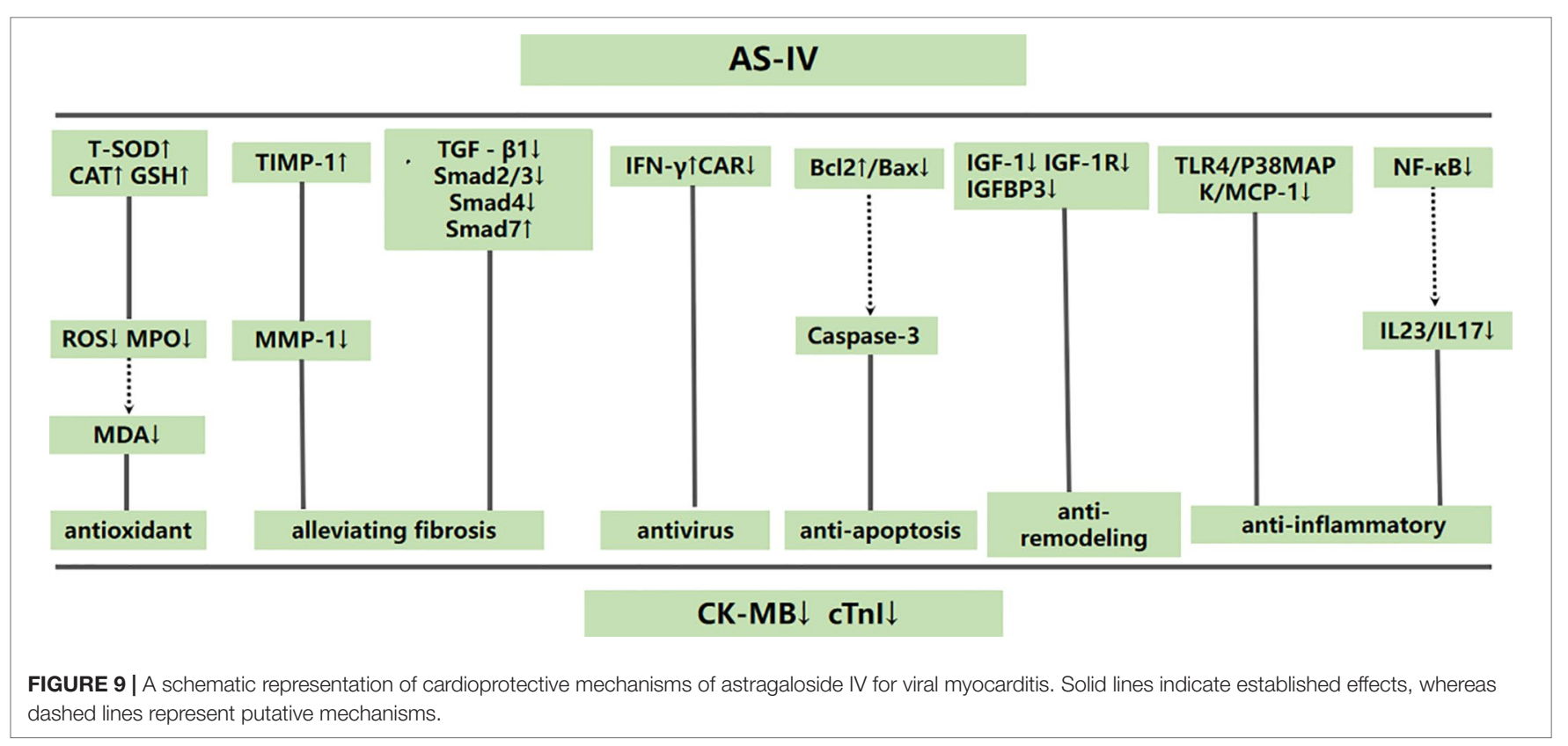

AS-IV on LVEDs of VM animals [ $\mathrm{n}=40$ SMD -2.88 95\% CI $(-3.83$ to -1.94$), \mathrm{P}<0.0001$; heterogeneity: $\left.\chi^{2}=0.83, \mathrm{I}^{2}=0 \%\right]$ (Figure 5).

\section{Cardiac Enzymes and/or Troponin}

One study (Gui et al., 2015) reported significant reduction CK-MB and CTnI levels of animals with VM treated with AS-IV $(\mathrm{P}<0.05)$.

\section{Cardioprotective Mechanisms}

Two studies (Wang and Li, 2007; Luo et al., 2008) reported a significant increase in T-SOD of VM animals by AS-IV, compared with the control group [ $\mathrm{n}=36 \mathrm{MD} 24.8195 \% \mathrm{CI}$ (19.88 to 29.73), $\mathrm{P}<0.0001$; heterogeneity: $\chi^{2}=0.26, \mathrm{I}^{2}=0 \%$ ] (Figure 6). Two studies (Zhang et al., 2003; Luo et al., 2010) focused on AS-IVinduced reduction of apoptosis index [n $=44$ SMD -3.09 95\% CI (-4.03 to -2.15$), \mathrm{P}<0.0001$; heterogeneity: $\left.\chi^{2}=1.76, \mathrm{I}^{2}=43 \%\right]$ (Figure 7), two (Wang and Li, 2007; Luo et al., 2008) on AS-IVinduced increase in GSH [n = 36 SMD 3.07 95\% CI (1.98 to 4.16), $\mathrm{P}<0.0001$; heterogeneity: $\chi^{2}=1.94, \mathrm{I}^{2}=49 \%$ ] (Figure 8), two (Zhou et al., 2012; Gui et al., 2015) on AS-IV-mediated reduction of TNF- $\alpha(P<0.05)$, one (Wang and Li, 2007) on reduction of ROS levels by AS-IV, one (Luo et al., 2010) on AS-IV-mediated increase in $\mathrm{Bcl}-2(\mathrm{P}<0.05)$ and reduction of $\mathrm{Bax}(\mathrm{P}<0.05)$, one
(Gui et al., 2015) on AS-IV-triggered NF-kB $(\mathrm{P}<0.05)$, and one (Gui et al., 2015) on reduction of IL-6 by AS-IV (P < 0.05). A schematic representation of the cardioprotective mechanisms of AS-IV is summarized in Figure 9.

\section{DISCUSSION}

\section{Summary of Evidence}

To our knowledge, this is the first preclinical systematic review (including 15 in vivo studies on a total of 577 animals) focused on evaluating the efficacy of AS-IV against VM and its potential mechanisms of action. Our findings clearly support cardioprotective effects of AS-IV in animal models of VM, mainly through improving anti-remodeling of the myocardium, anti-inflammatory, anti-apoptosis, anti-virus, antioxidant, and alleviation of myocardial fibrosis.

\section{Methodological Considerations}

Assessing of the validity of the included studies is a key component of a systematic review, and subsequently affects its analysis, interpretation and conclusions. Based on the Cochrane Handbook for Systematic Reviews of Interventions (Higgins and 
Green, 2011), the validity of an individual study should include two dimensions: external validity and internal validity. The former refers to whether the study is asking the appropriate research questions and the latter is in a manner free from the risk of bias of the included studies. In the present study, an appropriate research question was initially set up to evaluate the efficacy and possible mechanism of AS-IV in the treatment of the animals with VM using preclinical systematic review method. When assessments of methodological quality of included preclinical studies, two main risk of bias tools have been developed, including the Collaborative Approach to Meta-Analysis and Review of Animal Data from Experimental Studies (CAMARADES) 10-item checklist (Van et al., 2007) and the SYstematic Review Centre for Laboratory animal Experimentation (SYRCLE)'s Risk of Bias tool (Hooijmans et al., 2014). The CAMARADES 10-item checklist is specific for stroke and neuroprotective study on animal model while the SYRCLE's Risk of Bias tool is extensively used to assess the methodological quality of animal studies. Here, we selected the SYRCLE's Risk of Bias tool to assess the quality of included study and the result indicated that the quality scores of all studies were generally moderate. Thus, we should treat the present results cautiously when applying the study's findings.

Accumulating evidence demonstrates that animal reports on biomedical research are often inadequate in many areas (Kilkenny et al., 2010). An earlier survey evaluated 271 randomly selected articles about animal reports by the National Centre for the Replacement, Refinement and Reduction of Animals in Research (NC3Rs). The results showed that 59\% articles overall declared the hypotheses or objectives of the study and the characteristics and number of animals used. Randomization (87\%) or blindness $(86 \%)$ was not used in the majority of the articles to reduce bias of animal selection and outcome assessments. In total, $70 \%$ of the articles used statistical methods to fully describe publications and present the results in an accurate manner (Kilkenny et al., 2009). Similarly, the articles selected for our analysis had methodological deficiencies, such as seldom using the blind method and allocation concealment. Therefore, concerns of poor experimental design and lack of transparent reports are raised, which can lead to failure of translation of preclinical animal research into clinical treatments for human diseases (Baker et al., 2014). Reporting guidelines develop clear and standard checklist formats to make animal reports more complete and transparent, and thus increase the value of animal reports in clinical practice (Moher et al., 2015). The Animal Research: Reporting In Vivo Experiments (ARRIVE) guidelines, which provide a checklist of 20 items to promote high-quality, comprehensive reporting, have been accepted by over 300 research journals worldwide (Kilkenny et al., 2010). We recommend adherence to these guidelines for the further design of animal studies. In particular, attention should be paid to calculation of sample size, blinding of outcome assessor and blinding of caregivers (Macleod et al., 2004). As a minimum requirement, harmonized animal research reporting principles (HARRP) has been set up based on a comparative analysis of the ARRIVE guidelines by the Gold Standard Publication Checklist (GSPC) and the Institute of Laboratory Animal Research (ILAR) Guidance. Thus, the HARRP provided the minimal reporting standards for animal-based research (Osborne et al., 2018).

\section{Implications}

This study confirmed that AS-IV induces a significant reduction in mortality, improves myocardial pathology, and suppresses expression of cardiac enzymes, supporting its utility as a therapeutic option for patients with VM. However, translation of preclinical experiments remains challenging in predicting the effectiveness of therapeutic strategies in clinical trials (Hackam, 2007). The differences in drug doses and time of drug administration between humans and animals are considered the main reasons underlying failure of translation of research from bench to patient bedside (Baker et al., 2014). It is important to note that the 15 articles included in this study are not consistent in terms of drug dose and time of drug administration. Thus, we recommend establishing drug concentration gradients and grouping administration times to establish the optimal treatment strategy. In view of the differences between clinical trials and animal research, further high-quality randomized controlled trials of AS-IV for VM are required.

Several methods are commonly used for establishing animal models of myocarditis. (1) CVB3 or encephalomyocarditis virus (EMCV) injection: the advantages of this model is use of virus, which is close to clinical practice and suitable for studying CVB3 replication. However, this method is associated with high mortality rates and requires high biosafety standards. (2) Reovirus or murine adenovirus type $1(\mathrm{MAV}-1)$ injection is a unique model of pediatric myocarditis but is not clinically relevant. (3) Trypanosoma cruzi (T. cruzi) infection model, which could recapitulate the course of Chagas disease, but requires a long period for study. (4) Immunization with a-myosin heavy chain $(\alpha-\mathrm{MyHC})$ or troponin I peptide and complete Freund's adjuvant (CFA). The limitation of this model, which is suitable for studying transition from myocarditis to DCM, is non-physiological disease induction. (5) T cell receptor specific to $a-\mathrm{MyHC}$ (TCR-M) transgenic mice. The advantages of this model are biosafety and suitability for studying the pathophysiology of heart-specific T cells. However, the lack of non-specific $\mathrm{T}$ cells presents a drawback. (6) Programmed cell death protein-1 (PD-1)/PD-1 ligand (PD-L1) deficiency. This model is suitable for investigating the side-effects of anti-PD-1/ PD-1L therapy but involves multiple organs. (7) A human leukocyte antigen (HLA-DQ8) transgenic mouse model is beneficial for studying cardiac antigen presentation but a humanmouse chimeric system is lacking at present (Błyszczuk, 2019). All the studies included in this review utilized a myocardial model induced by CVB3 injection. Since CVB3 is closely related to the pathogenesis of myocarditis in human, this method of modeling is rational. We therefore recommend application of CVB3 to establish models of VM.

\section{Mechanisms}

Based on the findings of the included studies, the possible mechanisms of AS-IV mediated cardiovascular protection are as follows: (1) antioxidant through increasing T-SOD, GSH-PX and CAT levels (Wang and Li, 2007; Luo et al., 2008) to inhibit release of ROS and MPO (Wang and Li, 2007), (2) anti-inflammatory activity through suppression the TLR4/P38MAPK/MCP-1 
(Wang and Li, 2007; Zhou et al., 2012) and IL23/IL17 pathways (Liu et al., 2014) and inhibition of NF- $\kappa B$ (Zhou et al., 2012) as well as MIF (Liu et al., 2009) and A20 (Liu et al., 2015), (3) alleviation of myocardial fibrosis through inhibiting TGF- $\beta 1$, Smad2/3 and Smad4 and enhancing Smad7 (Chen et al., 2011) and increasing TIMP-1to reduce MMP-1 release (Xiao et al., 2016), (4) prevention of viral spread through increasing IFN- $\gamma$ (Zhang et al., 2006) and decreasing CAR expression (Yu et al., 2005), (5) inhibition of apoptosis through enhancing the Bcl-2/ Bax ratio (Luo et al., 2010), (6) anti-remodeling of myocardium through suppressing the expression of IGF-1, IGF-1R and IGFBP3 (He and Li, 2011).

\section{CONCLUSION}

The collective research clearly demonstrates that AS-IV decreases mortality, inflammation of the myocardium, pathological score and cardiac enzyme activity and improves heart function.

\section{REFERENCES}

Błyszczuk, P. (2019). Myocarditis in humans and in experimental animal models. Front. Cardiovasc. Med. 6, 64. doi: 10.3389/fcvm.2019.00064

Baker, D., Lidster, K., Sottomayor, A., and Amor, S. (2014). Two years later: journals are not yet enforcing the ARRIVE guidelines on reporting standards for pre-clinical animal studies. PLoS Biol. 12, e1001756. doi: 10.1371/journal. pbio. 1001756

Caforio, A. L., Mahon, N. J., Tona, F., and McKenna, W. J. (2002). Circulating cardiac autoantibodies in dilated cardiomyopathy and myocarditis: pathogenetic and clinical significance. Eur. J. Heart Fail. 4, 411-417. doi: 10.1016/s1388-9842(02)00010-7

Chen, P., Xie, Y. Q., Shen, E., Li, G. G., Yu, Y., Zhang, C. B., et al. (2011). Astragaloside IV attenuates myocardial fibrosis by inhibiting TGF- $\beta 1$ signaling in coxsackievirus B3-induced cardiomyopathy. Eur. J. Pharmacol. 658, 168-174. doi: 10.1016/j.ejphar.2011.02.040

Cooper, L. T., Keren, A., Sliwa, K., Matsumori, A., and Mensah, G. A. (2014). The global burden of myocarditis: part 1: a systematic literature review for the Global Burden of Diseases, Injuries, and Risk Factors 2010 study. Glob. Heart 9 , 121-129. doi: 10.1016/j.gheart.2014.01.007

Dennert, R., Crijns, H. J., and Heymans, S. (2008). Acute viral myocarditis. Eur. Heart J. 29, 2073-2082. doi: 10.1093/eurheartj/ehn296

GBD 2015 Disease and Injury Incidence and Prevalence Collaborators. (2016). Global, regional, and national incidence, prevalence, and years lived with disability for 310 diseases and injuries, 1990-2015: a systematic analysis for the Global Burden of Disease Study 2015. Lancet 388, 1545-1602. doi: 10.1016/ S0140-6736(16)31678-6

Gui, J., Chen, R. Z., Xu, W., and Xiong, S. D. (2015). Remission of CVB3-induced myocarditis with Astragaloside IV treatment requires A20 (TNFAIP3) up-regulation. J. Cell. Mol. 19, 850-864. doi: 10.1111/jcmm.12459

Hackam, D. G. (2007). Translating animal research into clinical benefit. BMJ 334, 163-164. doi: 10.1136/bmj.39104.362951.80

He, C. Z., and Li, S. J. (2011). Effects of Astragaloside on IGF-1 and associated protein expression in mice with acute viral myocarditis. Chin. J. Contemp. Pediatr. 13, 751-754. doi: 10.1111/j.1600-0714.2011.01024.x

Higgins, J. P. T., and Green, S. (2011). Cochrane Handbook for Systematic Reviews of Interventions. Version 5.1.0. updated March 2011. The Cochrane Collaboration. Available at: http://handbook-5-1.cochrane.org

Hooijmans, C. R., Rovers, M. M., de Vries, R. B., Leenaars, M., Ritskes-Hoitinga, M., and Langendam, M. W. (2014). SYRCLE's risk of bias tool for animal studies. BMC Med. Res. Methodol. 26, 14. doi: 10.1186/1471-2288-14-43

Kühl, U., and Schultheiss, H. P. (2009). Viral myocarditis: diagnosis, aetiology and management.Drugs 69, 1287-1302.doi: 10.2165/00003495-200969100-00001
Moreover, AS-IV exerts potential cardioprotective function of in VM primarily via improving anti-remodeling of myocardium, anti-inflammatory, anti-apoptosis, anti-virus, antioxidant and alleviation of myocardial fibrosis. Thus, AS-IV is a cardioprotective recruit for further clinical trials on VM.

\section{AUTHOR CONTRIBUTIONS}

Study conception and design: ZZ, G-QZ, and YW. Acquisition and analysis of data: ZZ, Z-HW, QZ, and L-HD. Manuscript writing: ZZ, Z-HW, and G-QZ. All authors gave final approval.

\section{FUNDING}

This project was supported by the grant of National Natural Science Foundation of China (81473491/81573750/81173395/ H2902).
Kilkenny, C., Parsons, N., Kadyszewski, E., Festing, M. F., Cuthill, I. C., Fry, D., et al. (2009). Survey of the quality of experimental design, statistical analysis and reporting of research using animals. PLoS One 30, 4. doi: 10.1371/journal. pone. 0007824

Kilkenny, C., Browne, W. J., Cuthill, I. C., Emerson, M., and Altman, D. G. (2010). Improving bioscience research reporting: The ARRIVE guidelines for reporting animal research. J. Pharmacol. Pharmacother. 1, 94-99. doi: 10.4103/0976-500X.72351

Lawson, C. M. (2000). Evidence for mimicry by viral antigens in animal models of autoimmune disease including myocarditis. Cell. Mol. Life Sci. 57, 552-560. doi 10.1007/PL00000717

Liu, P.Y., Yu,X.H.,Li, S. J., andBian, J.L. (2009). Roleofmacrophagemigrationinhibitory factor in viral myocarditis and its change after treatment with Astragaloside. Pharm. Care Res. 9, 18-21. doi: 10.3969/j.issn.1671-2838.2009.01.004

Liu, D. L., Liu, H. Y., and Gan, S. L. (2014). Inhibition of viral myocarditis by Astragaloside through IL- 23/IL- 17 signaling pathway. J. Clin. Pediatr. 32, 570573. doi: 10.3969/j.issn.1000-3606.2014.06.018

Luo, Y. J., Li, S. J., and Liu, H. Y. (2008). Protective effect of astragaloside on viral myocarditis in mice with CVB3. Chin. J. Arterioscler. 16, 205-208. doi: 10.3969/j. issn.1007-3949.2008.03.010

Luo, Y. J., Den, H., Li, S. J., and Du, Z. J. (2010). Anti-apoptosis effect and mechanism of astragaloside on viral myocarditis in Balb/C mice with CVB3. J. Univ. South China 38, 328-331. doi: 10.3969/j.issn.2095-1116.2010.03.008

Macleod, M. R., Collins, T. O., Howells, D. W., and Donnan, G. A. (2004). Pooling of animal experimental data reveals influence of study design and publication bias. Stroke 35, 1203-1208. doi: 10.1161/01.STR.0000125719.25853.20

Mason, J. W. (2003). Myocarditis and dilated cardiomyopathy: an inflammatory link. Cardiovasc. Res. 60, 5-10. doi: 10.1016/s0008-6363(03)00437-1

McManus, B. M., Chow, L. H., Wilson, J. E., Anderson, D. R., Gulizia, J. M., Gauntt, C. J., et al. (1993). Direct myocardial injury by enterovirus: a central role in the evolution of murine myocarditis. Clin. Immunol. Immunopathol. 68, 159-169. doi: 10.1006/clin.1993.1113

Moher, D., Avey, M., Antes, G., and Altman, D. G. (2015). The National Institutes of Health and guidance for reporting preclinical research. BMC Med. 13, 34. doi: 10.1186/s12916-015-0284-9

Osborne, N., Avey, M. T., Anestidou, L., Ritskes-Hoitinga, M., and Griffin, G. (2018). Improving animal research reporting standards: HARRP, the first step of a unified approach by ICLAS to improve animal research reporting standards worldwide. EMBO Rep. 19, e46069. doi: 10.15252/embr.201846069

Pollack, A., Kontorovich, A. R., Fuster, V., and Dec, G. W. (2015). Viral myocarditisdiagnosis, treatment options, and current controversies. Nat. Rev. Cardiol. 12 , 670-680. doi: 10.1038/nrcardio.2015.108 
Ponikowski, P., Voors, A. A., Anker, S. D., Bueno, H., Cleland, J. G. F., Coats, A. J. S., et al. (2016). 2016 ESC guidelines for the diagnosis and treatment of acute and chronic heart failure. Rev. Esp. Cardiol. (Engl. Ed.) 69, 1167. doi: 10.1016/j.rec.2016.11.005

Ren, S., Zhang, H., Mu, Y., Sun, M., and Liu, P. (2013). Pharmacological effects of Astragaloside IV: a literature review. J. Tradit. Chin. Med. 33, 413-416. doi: 10.1016/s0254-6272(13)60189-2

Sena, E. S., Currie, G. L., McCann, S. K., Macleod, M. R., and Howells, D. W. (2014). Systematic reviews and meta-analysis of preclinical studies: why perform them and how to appraise them critically. J. Cereb. Blood Flow Metab. 34, 737-742. doi: $10.1038 /$ jcbfm.2014.28

Stewart, L. A., Clarke, M., Rovers, M., Riley, R. D., Simmonds, M., and Stewart, G. (2015). Preferred reporting items for systematic review and metaanalyses of individual participant data: the PRISMA-IPD Statement. JAMA 313, 16571665. doi: 10.1001/jama.2015.3656

Tian, L., He, C. Z., and Li, S. J. (2015). Effect of astragaloside on TL1A expression in viral myocarditis. Zhong Nan Da Xue Xue Bao Yi Xue Ban 40, 150-157. doi: 10.11817/j.issn.1672-7347.2015.02.006

Van, d. W. H. B., Sena, E. S., Donnan, G. A., Howells, D. W., Macleod, M. R., et al. (2007). Hypothermia in animal models of acute ischaemic stroke: a systematic review and meta-analysis. Brain 130, 3063-3074. doi: 10.1093/brain/awm083

Wang, M. H., and Li, S. J. (2007). The antioxidant effect of Astragaloside on coxsackievirus B3-induced murine myocarditis. J. Clin. Pediatr. 25, 825-827. doi: 10.3969/j.issn.1000-3606.2007.10.008

Wang, L. L. (2012). Effect of Astragaloside on expression of TLR4, P38MAPK and MCP-1 in mice with viral myocarditis. Available online at: http://www. wanfangdata.com.cn/details/detail.do?_type=degree\&id=Y2237637

Xiao, Y. F., Liu, S., Li, W. Y., and Liu, X. L. (2016). The influence of Astragalus Saponin on TIMP-1 and MMP-1 in viral myocarditis model mice induced by CVB3. Guid. J. Tradit. Chin. Med. Pharm. 22, 22-28. doi: 10.13862/j.cnki. cn43-1446/r.2016.23.006

Yu, X. H., Zhang, X. G., Wang, S. J., Zhao, G., Chen, R. Z., Yang, Y. Z., et al. (2005). Effect of Astragaloside on gene expression of Coxsackie-Adenovirus receptor in viral myocarditic mice. J. Appl. Clin. Pediatr. 20, 215-217. doi: 10.3969/j. issn.1003-515X.2005.03.012

Zhang, Z. C., Yang, Y. Z., Li, S. J., and Chen, H. Z. (2003). Effect of astragaloside on myocardial fibrosis in viral myocarditic mice. Chin. J. New Drugs Clin. Rem. 22, 515. doi: 10.3969/j.issn.1007-7669.2003.09.001

Zhang, Y. Y., Zhu, H. Y., Huang, C. G., Cui, X. L., Gao, Y. J., Huang, Y., et al. (2006). Astragaloside IV exerts antiviral effects against Coxsackievirus B3 by upregulating interferon- $\gamma$. J. Cardiovasc. Pharmacol. 47, 190-195. doi: 10.1097/01.fjc.0000199683.43448.64

Zhou, X., Liu, C. M., Hu, A. N., Du, Z. Q., Wang, L. L., Wu, Y., et al. (2012). Effect of astragaloside on expression of TLR4, NF- $\kappa B$ and TNF- $\alpha$ in mice with viral myocarditis. Chin. J. Clinicians 6, 3659-3662. doi: $10.3877 / \mathrm{cma}$.J.I ssn.1674-0785.2012.13.095

Conflict of Interest: The authors declare that the research was conducted in the absence of any commercial or financial relationships that could be construed as a potential conflict of interest.

Copyright (c) 2019 Zhuang, Wang, Deng, Zheng, Zheng and Wang. This is an openaccess article distributed under the terms of the Creative Commons Attribution License (CC BY). The use, distribution or reproduction in other forums is permitted, provided the original author(s) and the copyright owner(s) are credited and that the original publication in this journal is cited, in accordance with accepted academic practice. No use, distribution or reproduction is permitted which does not comply with these terms. 